\title{
Breast Ultrasound during Lactation: Benign and Malignant Lesions
}

\author{
Nuray Haliloglu Evren Ustuner Esra Ozkavukcu \\ Department of Radiology, Ankara University Faculty of Medicine, Ankara, Turkey
}

\section{Keywords}

Lactation · Breast · Ultrasound · Galactocele · Lactating adenoma

\begin{abstract}
Background: Structural changes during lactation make breast physical examination difficult. When breast problems occur, patients are often referred for an ultrasound (US) scan. Most breast lesions diagnosed in these patients are benign, but the diagnosis of breast cancer is a challenge. We aim to demonstrate the spectrum of US imaging findings in lactating women. Methods: 77 breastfeeding patients who underwent breast US in our department between February 2012 and March 2017 were evaluated. Patients' electronic medical records were reviewed for the presenting complaint, US reports, pathology results if available, and clinical/radiologic follow-up. All examinations were performed by 2 radiologists. Results: 28 of the 77 patients had normal US findings. Cysts were seen in 16 patients. 4 patients had stable fibroadenomas. 6 patients had US imaging findings suggestive of mastitis, 5 patients had galactoceles, 1 patient had an abscess, and 1 patient had unilateral hypertrophy without any accompanying lesion. In 13 patients, BI-RADS 3 solid masses were diagnosed. Invasive breast cancer was diagnosed in 3 patients. Conclusion: US can demonstrate or exclude a true mass against the background of a nodular breast parenchyma. Radiologists must be aware of malignant US features to avoid delays in the diagnosis of pregnancy-associated breast cancer.
\end{abstract}

(C) 2018 S. Karger GmbH, Freiburg

\section{KARGER}

(c) 2018 S. Karger GmbH, Freiburg

E-Mail karger@karger.com

www.karger.com/brc

\section{Introduction}

Following lobular growth and cellular proliferation during pregnancy, the breast transitions to a secretory state during lactation. Alterations in serum estrogen, progesterone, and prolactin levels are mainly responsible for these structural changes $[1,2]$. Due to the increased size, nodularity, and sensitivity of the breasts during lactation, physical examination is usually a challenge. Ultrasound (US) is almost always the first imaging step in lactating women to confirm the presence of a breast lump and to differentiate between cystic and solid lesions. Although some lesions may increase in size, most of the breast lesions detected during lactation are benign [3]. On the other hand, pregnancy-associated breast cancer is a devastating diagnosis with a poor prognosis and a negative impact on both the mother's and the baby's quality of life. Therefore, it is obligatory to be aware of breast lesions seen during lactation, and to be vigilant about malignant features. The aim of this study is to demonstrate the spectrum of breast US imaging findings in breastfeeding women.

\section{Patients and Methods}

The Institutional Ethics Committee approved this retrospective study protocol and waived informed consent.

Our study included 77 actively breastfeeding patients who underwent breast US in our department between February 2012 and March 2017. The mean age of the patients was 31.6 years (range 20-46 years). Patients' electronic medical records were reviewed for the presenting complaint, breast US reports, pathology results if available, and clinical/radiologic follow-up. All examinations were performed by 2 radiologists with more than 10 years' experience in US imaging (US systems: Aplio XG, Toshiba, Tokyo, Japan; LOGIQ 7, GE Healthcare, Milwaukee, WI, USA). 


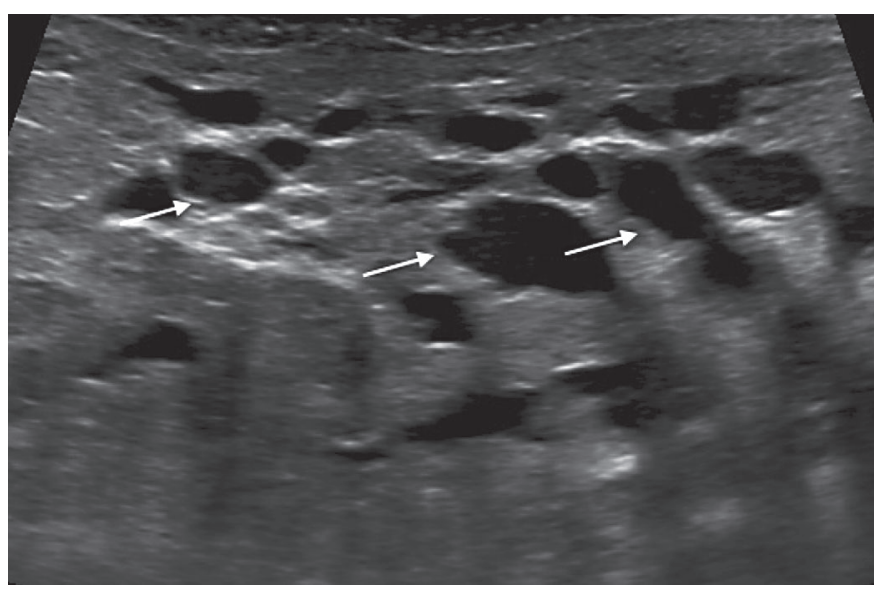

Fig. 1. Normal sonographic appearance of a lactating breast. Parenchyma is slightly hyperechoic and ducts are dilated (arrows).

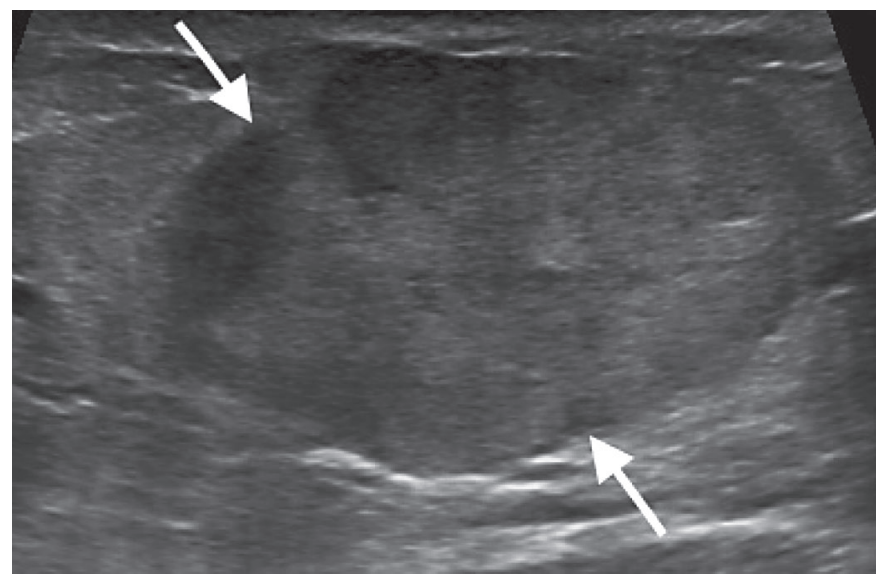

Fig. 3. Ultrasound image of a 35 -year-old lactating woman who presented with a painless palpable mass which appeared during pregnancy and progressed during lactation. The mass has distinct but slightly lobulated margins (arrows). Histopathologic analysis revealed benign findings indicating lactating adenoma.

A 'Breast Imaging Reporting and Data System' (BI-RADS) category was assigned to all US examinations on the basis of standardized descriptive criteria published by the American College of Radiology [4].

Hyperechoic breast parenchyma and prominent ducts were considered as physiologic lactational changes.

Well-circumscribed anechoic masses with smooth walls and posterior acoustic enhancement were simple cysts. Cysts containing internal low-level echoes or septae, or lacking the classical features of simple cysts were called complicated cysts.

US features of mastitis were mild skin thickening and parenchymal edema. An abscess was defined as a thick-walled anechoic or hypoechoic mass with posterior acoustic enhancement, with or without internal gas and debris.

Galactoceles were defined as round or oval shaped, anechoic or hypoechoic, well-circumscribed masses, with or without internal echogenicity or a fat-fluid level, and without internal vascularity.

The size, shape, margins, and echogenicity of solid lesions were noted, and

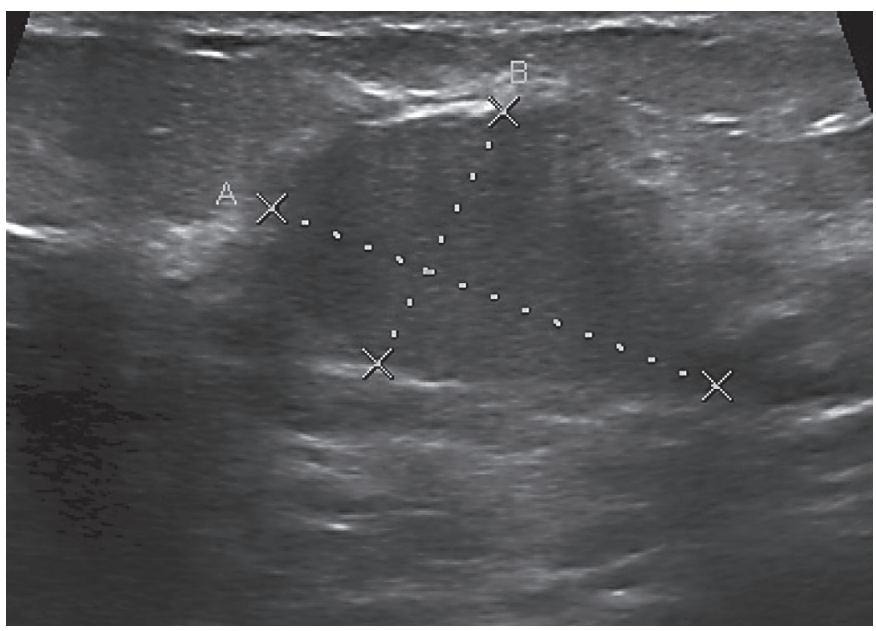

Fig. 2. Ultrasound image of a breastfeeding woman who presented with a painless palpable mass showing a large, oval-shaped, hypoechoic, well-circumscribed mass with posterior acoustic enhancement. The patient was followedup for 1 year and the size of the mass regressed.

taller-than-wide hypoechoic lesions with irregular shape, ill-defined/microlobulated margins, and inflammatory changes (skin thickening, parenchymal edema) not improving with therapy were considered highly suspicious for malignancy.

\section{Results}

The time interval between delivery and the initial breast US examination ranged between 1 and 32 months. Indications for breast US were palpable mass in 25 patients, diffuse swelling, pain, and/or tenderness in 29 patients, palpable axillary mass in 5 patients, skin erythema in 4 patients, and bloody nipple discharge in 3 patients. 1 patient was admitted to our hospital for progression of an already existing nipple lesion and axillary lymphadenopathy. 10 of the 77 patients were asymptomatic and were referred to US imaging for follow-up of existing fibrocystic disease, or fibroadenomas, or for screening due to a family history of breast cancer.

Of the 77 patients, 28 (36\%) had normal US imaging findings with lactational changes in the breast parenchyma (BI-RADS 1) (fig. 1). Simple or complicated cysts were seen in 16 patients, among which only 1 lesion showed an increase in size compared to the pre-pregnancy US. 4 patients had stable fibroadenomas. 6 of the 77 patients had US imaging findings suggestive of mastitis, 5 patients had galactoceles (fig. 2), 1 patient had an abscess, and 1 patient had unilateral hypertrophy without any accompanying lesion (BI-RADS 2).

In $12(15.6 \%)$ of the 77 patients, BI-RADS 3 solid masses were diagnosed. 2 of these 12 patients were followed-up for 2 years and the lesions were stable. 6 of the 12 patients underwent biopsy, and the histopathologic diagnosis was lactating adenoma in 3 patients (fig. 3), benign proliferative changes in 2 patients, and granulomatous mastitis in 1 patient. 4 of the 12 patients with BI-RADS 3 lesions were lost to follow-up. 


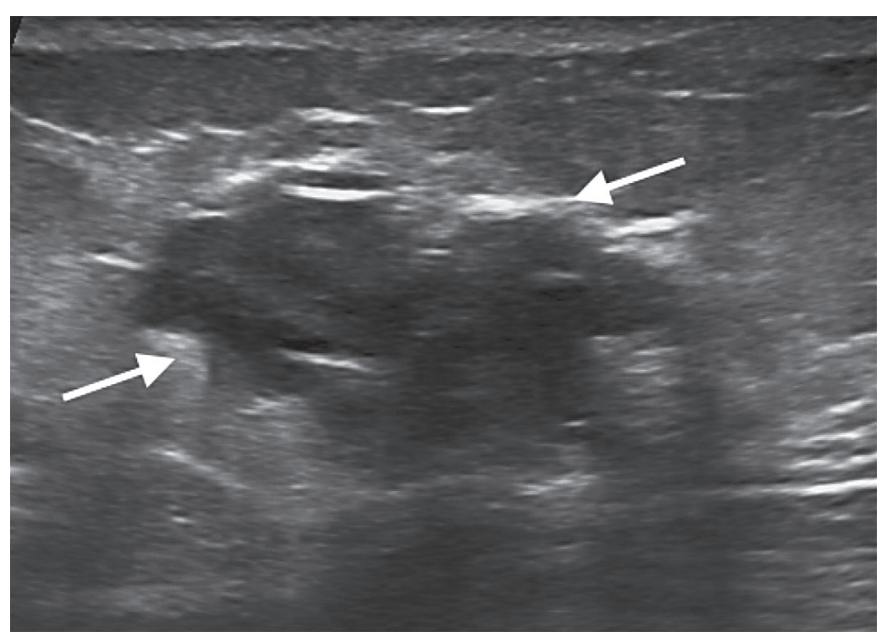

Fig. 4. Ultrasound revealed a hypoechoic mass with irregular margins in a 33-year-old lactating woman presenting with a painless palpable mass (BIRADS 4). Biopsy showed no signs of malignancy. The lesion was downgraded to BI-RADS 3.

Table 1. Ultrasound imaging findings

\begin{tabular}{ll}
\hline Ultrasound imaging findings & Patients, n (other findings) \\
\hline $\begin{array}{l}\text { Normal US imaging findings with } \\
\quad \text { lactational changes }\end{array}$ & 28 \\
Simple or complicated cysts & 16 \\
Pre-existing fibroadenomas & 4 \\
Galactocele & 5 \\
Mastitis (no abscess) & 6 \\
Abscess & 1 \\
Unilateral hypertrophy & 1 \\
BI-RADS 3 solid mass & 12 (6 biopsy-proven benign lesions, 2 \\
& lesions stable over 2 years of follow-up, \\
BI-RADS 4 solid mass & 4 lesions lost to follow-up) \\
& 1 (biopsy-proven benign lesion down- \\
Invasive breast cancer & graded to BI-RADS 3) \\
& 3 \\
\hline
\end{tabular}

US imaging demonstrated a hypoechoic solid mass with irregular borders (BI-RADS 4) in 1 patient presenting with a painless palpable mass (fig. 4). Histopathologic analysis revealed benign proliferative changes and periductal inflammation without malignant features. The lesion was then downgraded to BI-RADS 3. The patient was followed-up for 1 year and the mass almost completely regressed after the cessation of lactation.

Invasive breast cancer was diagnosed in 3 (3.9\%) of the $77 \mathrm{pa}-$ tients. 1 of these 3 patients had clinical findings suggestive of mastitis which did not respond to antibiotic treatment. US demonstrated diffuse skin thickening, parenchymal edema, undefined hypoechoic areas, and clustered microcalcifications (fig. 5). The patient underwent US-guided biopsy. 1 patient presented with a palpable mass which was categorized as BI-RADS 5. 1 patient had

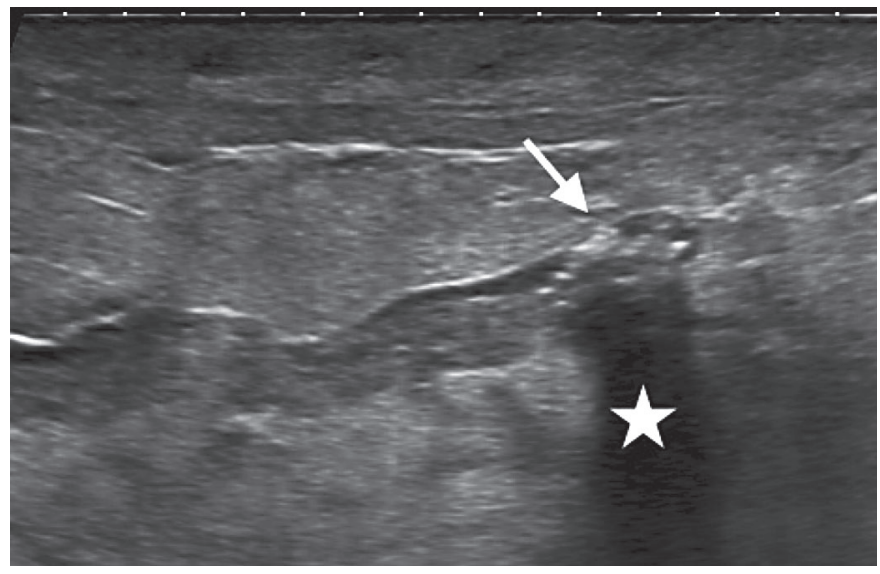

Fig. 5. Ultrasound image of a 33-year-old lactating patient with invasive breast cancer demonstrating clustered ductal microcalcifications (arrow) and their posterior acoustic shadowing (asterisk). The patient underwent chemotherapy and surgery but bone metastasis occurred within 2 years.

skin changes which progressed during pregnancy and lactation. She had diffuse inflammatory changes, intraductal and periductal solid masses, and axillary lymphadenopathy on US. Histopathologic analysis revealed invasive ductal carcinoma in all 3 of these patients. Results are summarized in table 1.

\section{Discussion}

Many breastfeeding women experience breast symptoms including pain, tenderness, firmness, and palpable lumps. Thanks to the increasing breast cancer awareness, these patients are usually referred for further examination. The lactating breast is under the influence of circulating hormones which lead to glandular proliferation, ductal dilatation, and stromal involution. Hence, the physical examination of the lactating breasts is difficult, and radiologic evaluation is usually necessary [5]. It has been suggested that, regardless of the lactational or gestational status, for symptomatic women younger than 30 years of age, US should be the first-line imaging and mammography should be saved for patients with indeterminate or suspicious lesions on US scans. US is quite successful in demonstrating true masses as well as normal breast parenchyma which may show palpable nodularity during lactation. When necessary, mammography can be performed just after breastfeeding to avoid high-density parenchyma related to retained milk products $[1,5]$. Magnetic resonance imaging findings may be altered due to physiologic changes but remains an efficient technique for the detection and characterization of breast lesions during lactation. There is no reported risk for the use of intravenous gadolinium chelates during lactation; however, breastfeeding may be suspended for $24 \mathrm{~h}$ after contrast medium administration [6]. 
Lactating breast parenchyma shows diffuse hyperechogenicity, dilated ducts, and increased vascularity on US scans [1]. Our study results are consistent with previous data from the relevant literature showing that the majority of breast masses detected during lactation are benign and in part unique to this period of life $[1,7]$. The sonographic appearance of cysts and fibroadenomas is similar to that seen in non-lactating patients, which is widely known. Galactoceles are the most common palpable masses in lactating patients. These are cystic masses with posterior acoustic shadowing and variable internal echonicity depending on their fat, protein, and water content, but vascularity should never be present within a galactocele [2, 8]. Lactating adenomas are fibroadenoma-like masses on US scan; however, microlobulations are more frequent and hypervascularity is usually seen on Doppler US. Lactating adenomas may increase in size during lactation. Spontaneous regression of the mass after cessation of lactation can be another diagnostic criterion, although some lesions may not resolve and surgical excision may be necessary. Lactating adenomas also differ from fibroadenomas with their unique histologic features. US-guided biopsy is usually encouraged despite the benign US features [2,9].

Patients with puerperal mastitis usually present with edema and erythema of the breast with pain, tenderness, and fever. It is a clinical diagnosis, and US should be saved for patients with poor response to antibiotic therapy or if an abscess is suspected. US features of mastitis can be limited to skin thickening and parenchymal edema, but sometimes mass-like hypoechoic lesions with indistinct borders and peripheral hypervascularity can be seen [2]. These lesions may appear stiff on elastography studies mimicking malignancy. Nevertheless, a mature abscess, which is usually seen as a thick-walled hypoechoic mass with posterior acoustic enhancement and some degree of internal echogenicity, would show a soft center and a stiff thick outer rim on elastography [10]. As well as being a useful diagnostic tool for breast abscess, US also provides a practical guide for catheter drainage, which is a safe, well-tolerated, and cost-effective treatment procedure allowing patients to continue breastfeeding [11]. Granulomatous mastitis is another challenging diagnosis occurring during or shortly after lactation. US features of granulomatous mastitis include an ill-defined, hypoechoic, heterogenous mass with internal tubular structures, which may extend outside the mass [12].

Pregnancy-associated breast cancer is defined as breast cancer diagnosed during pregnancy or in the first postpartum year. The average age of the patients is $32-38$ years [13]. It is a rare but important diagnosis, and US is highly sensitive in the detection of these malignant lesions. Patients with persisting palpable masses should undergo US imaging, and in the case of any suspicious finding, further evaluation with US-guided biopsy should be performed to avoid a delay in diagnosis [14]. Pregnancy-associated breast cancer usually presents with a palpable mass that is larger and in a more advanced stage than those seen in non-pregnant or non-lactating patients $[5,7]$. It has been reported that the frequency is approximately 1 in 3,000 pregnancies, but due to the increasing trend for women to delay motherhood, the frequency of this cancer will probably increase in the next years [15]. In our study group, pregnancy-associated breast cancer was found at a rate of $3.9 \%$. This does not reflect the true incidence of the cancer as we did not perform US in all lactating patients. Nevertheless, this rate gives an idea about the frequency of breast cancer in lactating patients with breast symptoms and should not be underestimated.

The sensitivity of US in the diagnosis of pregnancy-associated breast cancer is reported to be between 93 and $100 \%$ with a negative predictive value of $100 \%$ in different studies. In the literature, malignant masses are usually described as predominantly hypoechoic lesions with an irregular shape and ill-defined margins [1517]. Posterior acoustic enhancement, which is usually seen in benign lesions, is also reported as a sonographic finding of pregnancy-associated breast cancer. Necrotic or cystic degeneration within the mass may produce posterior enhancement and may reflect the rapid growth of the tumor. Although malignant lesions are usually 'taller than wide', it has been reported that pregnancy-associated breast cancer may present with parallel orientation [16]. Any new solid or complex cystic palpable mass identified on US during lactation that does not resolve within 2 weeks warrants biopsy even if it has a benign US appearance. Biopsy-proven benign lesions can be followed-up carefully during and after lactation as performed in non-lactating women $[2,15,17]$.

Although there is no certain histologic type of pregnancy-associated breast cancer, inflammatory carcinoma should also be kept in mind when there is no response to antibiotic therapy in patients with symptoms suggesting mastitis as seen in 1 of our patients.

In conclusion, breast examination deserves careful attention during lactation. Both, clinicians and radiologists must be aware of possible breast lesions which are sometimes unique to lactation. Most of the lesions seen in these patients are benign, and US can successfully demonstrate or exclude a true mass against the background of a nodular parenchyma. In the evaluation of lactating patients, radiologists must be aware of malignant US features to avoid delays in the diagnosis of pregnancy-associated breast cancer.

\section{Disclosure Statement}

There is no financial support or conflict of interest to declare.

\section{References}

Sabate JM, Clotet M, Torrubia S, et al: Radiologic evaluation of breast disorders related to pregnancy and lactation. Radiographics 2007;27:S101-S124.

2 Vashi R, Hooley R, Butler R, Geisel J, Philpotts L: Breast imaging of the pregnant and lactating patient: physiologic changes and common benign entities. Am J Roentgenol 2013;200:329-336

3 Langer A, Mohallem M, Berment H, et al: Breast lumps in pregnant women. Diagn Interv Imaging 2015;96:1077-1087.

4 American College of Radiology: BI-RADS: ultrasound, ed 1; in: Breast Imaging Reporting and Data System: BI-RADS Atlas. Reston, VA, American College of Radiology, 2003.

5 Robbins J, Jeffries D, Roubidoux M, Helvie M: Accuracy of diagnostic mammography and breast ultrasound during pregnancy and lactation. Am J Roentgenol 2011;196: $716-722$. 
6 Boivin G, de Korvin B, Marion J, Duvauferrier R: Is a breast MRI possible and indicated in case of suspicion of breast cancer during lactation? Diagn Interv Imaging 2012;93:823-827.

7 Hosny IA, Eldin LAS, Elghawabi HS: Radiological evaluation of palpable breast masses during pregnancy and lactation. Egypt J Radiol Nucl Med 2011;42:267273.

8 Holanda AAR, Goncalves AKS, Medeiros RD, Oliveira AMG, Maranhao TMO: Ultrasound findings of the physiological changes and most common breast diseases during pregnancy and lactation. Radiol Bras 2016;49:389-396.

-9 Parnes AN, Akalin A, Quinlan RM, Vijayaraghavan GR: AIRP best cases in radiologic-pathologic correlation lactating adenoma. Radiographics 2013;33:455459.
Sousaris N, Barr RG: Sonographic elastography of mastitis. J Ultrasound Med 2016;35:1791-1797.

11 Falco G, Foroni M, Castagnetti F, et al: Ultrasoundguided percutaneous catheter drainage of large breast abscesses in lactating women: how to preserve breastfeeding safely. Breastfeeding Med 2016;11: 555-556.

12 Freeman CM, Xia BT, Wilson GC, et al: Idiopathic granulomatous mastitis: a diagnostic and therapeutic challenge. Am J Surg 2017;214:701-706.

13 Molckovsky A, Madarnas Y: Breast cancer in pregnancy: a literature review. Breast Cancer Res Treat 2008; 108:333-338.
4 Vashi R, Hooley R, Butler R, Geisel J, Philpotts L: Breast imaging of the pregnant and lactating patient: imaging modalities and pregnancy-associated breast cancer. Am J Roentgenol 2013;200:321-328.

5 Kakoulidis I, Skagias L, Politi E: Pregnancy associated breast cancer (PABC): aspects in diagnosis. Breast Dis 2015;35:157-166.

16 Ahn BY, Kim HH, Moon WK, et al: Pregnancy- and lactation-associated breast cancer: mammographic and sonographic findings. Ultrasound Med 2003;22:491497.

7 Ayyappan AP, Kulkarni S, Crystal P: Pregnancy-associated breast cancer: spectrum of imaging appearances. Brit J Radiol 2010;83:529-534. 